\title{
Adaptação e avaliação da consistência interna do Postpartum Thoughts and Behavior Checklist
}

\author{
Adaptation and internal consistency evaluation of the \\ Postpartum Thoughts and Behavior Checklist
}

Laila da Camara Lima Kurtinaitis', Carla Fonseca Zambaldi², Tarcísio Gomes Dutra', Marília Suzi Pereira dos Santos ${ }^{1}$, Amaury Cantilino da Silva Júnior ${ }^{3}$, Karine Porpino Viana ${ }^{4}$, Everto Botelho Sougey ${ }^{5}$

\section{RESUMO}

Objetivo: Traduzir, adaptar e avaliar a aplicabilidade, além de obter dados acerca da consistência interna do Postpartum Thoughts and Behavior Checklist. Esse instrumento foi elaborado especificamente para uso durante o pós-parto e identifica a presença e o conteúdo dos pensamentos intrusivos em relação ao bebê, assim como as estratégias de neutralização utilizadas após esses pensamentos. Método: A tradução do instrumento para o português foi feita por dois profissionais de saúde mental bilíngues. Posteriormente, houve uma retrotradução realizada por dois professores de inglês. A versão retrotraduzida foi avaliada pelo autor da escala e aplicada em 91 puérperas usuárias de um serviço da rede pública de saúde. Resultados: A versão traduzida do Postpartum Thoughts and Behavior Checklist foi considerada equivalente ao instrumento original em inglês. As participantes não apresentaram dificuldades na compreensão dos itens do checklist. Sua consistência interna, medida pelo alfa de Cronbach, foi de 0,822. Conclusão: A versão em português do Postpartum Thoughts and Behavior Checklist pode ser considerada adequada para o uso na população estudada para avaliar pensamentos obsessivos em relação ao bebê durante o puerpério e possíveis estratégias de neutralização desses pensamentos. O checklist pode ainda trazer contribuições significativas para a prática clínica.

\begin{abstract}
Objective: To translate, adapt, evaluate the applicability, and to obtain data regarding the internal consistency of Postpartum Thoughts and Behavior Checklist. This checklist is a specific instrument for postpartum which identify the presence and the content of intrusive thoughts related to newborn as well as the neutralization strategies used after these thoughts. Method: The translation of the instrument into Portuguese was carried out by two bilingual mental health professionals. Afterwards, a back translation was performed by two English teachers. The back
\end{abstract}

1 Universidade Federal de Pernambuco (UFPE), Programa de Pós-graduação em Neuropsiquiatria e Ciências do Comportamento. 2 Universidade Federal de Pernambuco (UFPE), Programa de Pós-graduação em Neuropsiquiatria e Ciências do Comportamento e Programa de Saúde Mental da Mulher.

3 Universidade Federal de Pernambuco (UFPE), Programa de Saúde Mental da Mulher.

4 Universidade Federal de Pernambuco (UFPE).

5 Universidade Federal de Pernambuco (UFPE), Departamento de Neuropsiquiatria e Ciências do Comportamento. 


\section{Keywords}

Postpartum, obsessive behavior, compulsive behavior. translation version was evaluated by the author of the scale and applied in 91 new mothers who were users of health public service. Results: The translated version of Postpartum Thoughts and Behavior Checklist was considered equivalent to the original instrument in English. The participants did not show difficulties to understand the checklist items. The internal consistency, measured through Cronbach's alpha, was 0,822. Conclusion: The Portuguese version of Postpartum Thoughts and Behavior Checklist could be considered appropriated for using in studied population in order to evaluate obsessive thoughts related to newborn during postpartum and possible strategies to neutralize these thoughts. The checklist could also bring significant contributions to the clinical practice.

\section{INTRODUÇÃO}

Apesar de a gestação ser considerada por muitos como um período de bem-estar emocional e de se esperar que o nascimento da criança seja um momento muito especial na vida da mulher, o puerpério consiste, para as mulheres, em uma fase de transição extremamente importante para o desenvolvimento de sua personalidade. É um período transitório biologicamente determinado, no qual se processam mudanças metabólicas concretas, ligadas a perspectivas de mudanças também no âmbito social. Tais mudanças implicam novas adaptações e reajustamento interpessoais e intrapsíquicos e, consequentemente, uma mudança de identidade'. Dessa maneira, a gestação e o puerpério precisam ser avaliados com atenção especial, pois eles podem interferir diretamente na saúde mental da mulher².

Os sintomas obsessivo-compulsivos têm sido frequentemente associados ao puerpério. O conteúdo dos pensamentos obsessivos apresentados pelas puérperas se assemelha ao das obsessões no Transtorno Obsessivo-Compulsivo (TOC). Podem ocorrer pensamentos obsessivos de conteúdo agressivo, contaminação, sexual, simetria, dúvida ou outros, todavia, é frequente que esses pensamentos ou imagens visuais envolvam o bebê. Por exemplo, a puérpera pode apresentar pensamento obsessivo envolvendo sufocamento ou acidentes com recém-nascido, ímpetos agressivos, ideias de contaminação com o bebê ou imagens de conteúdo sexual ${ }^{3-5}$. Os pensamentos obsessivos algumas vezes podem ocorrer acompanhados de sintomas compulsivos ${ }^{6}$, como compulsão por checagem, limpeza/lavagem ou outros rituais.

Um estudo realizado com mulheres da região metropolitana do Recife (PE) mostrou que 63\% das mulheres no puerpério apresentavam alguma obsessão e/ou compulsão7. Outro estudo, realizado com puérperas e seus companheiros, demonstrou que 89,4\% dos participantes tiveram, em algum momento, pensamentos estressantes sobre o bebê e que a maioria deles se utilizava de alguma estratégia para neutralizar esses pensamentos ${ }^{4}$. Dessa maneira, alguns autores afirmam que os pensamentos obsessivos e negativos durante o puerpério são comuns também em mulheres saudáveis ${ }^{8}$.
Alguns estudos têm demonstrado a necessidade de um maior esclarecimento e aprofundamento sobre os possíveis padrões de pensamento de mulheres no puerpério ${ }^{8,9}$. Foi observada ainda a pequena quantidade de instrumentos de medida psicométrica que se referem especificamente a esse período da vida das mulheres².

No Brasil, não há um instrumento delineado especificamente para avaliar os pensamentos obsessivos no puerpério. Para a medida de pensamentos obsessivos, a escala mais utilizada é a Yale-Brown Obsessive Compulsive Scale (Y-Bocs) ${ }^{10}$. Entretanto, esse instrumento não se refere aos pensamentos obsessivos e comportamentos compulsivos mais recorrentes no pós-parto que, em sua maioria, dizem respeito ao recém-nascido e seus cuidados.

Um pesquisador norte-americano desenvolveu uma escala que avalia especificamente os pensamentos obsessivos e comportamentos compulsivos apresentados no puerpério. Essa escala foi desenvolvida no idioma inglês e é denominada Postpartum Thougths and Behavior Checklist (PTBC). Ela consiste num checklist que identifica a presença e o conteúdo dos pensamentos intrusivos que ocorrem no pós-parto e as estratégias de neutralização utilizadas pelos pais após esses pensamentos.

O PTBC se divide em quatro sessões. Na primeira, o entrevistador define e normaliza a ocorrência dos pensamentos intrusivos e incômodos que podem ocorrer no puerpério. São dados exemplos de pensamentos intrusivos e os participantes são encorajados a discutir seus próprios pensamentos. Em seguida, há um checklist sobre as dificuldades existentes nos cuidados diários para com o bebê. A terceira sessão do instrumento contém um checklist com 35 pensamentos intrusivos do pós-parto. Esses itens foram categorizados em sete temas: a) sufocamento; b) pensamentos intrusivos de agressividade/prejuízo/violência; c) acidentes/ responsabilidade; d) pensamentos sobre perder o bebê; e) contaminação; f) obsessões sexuais; g) doenças ${ }^{3}$. A quarta sessão contém um checklist similar ao anterior, com 13 questões acerca do comportamento e estratégias mentais que os novos pais costumam utilizar para manejar os pensamentos intrusivos relacionados ao bebê ${ }^{11}$. 
O conhecimento mais aprofundado acerca dos pensamentos obsessivos e comportamentos compulsivos mais comuns no puerpério pode ser útil para um maior conhecimento sobre as cognições nesse período da vida da mulher e para a elaboração do planejamento terapêutico de mulheres com sintomas obsessivo-compulsivos no puerpério. Além disso, a utilização do PTBC permitirá a comparação de pesquisas realizadas no Brasil a dados obtidos em outros países.

Diante da necessidade de um instrumento que se refira aos pensamentos obsessivos característicos do pós-parto, o presente estudo pretende traduzir, adaptar e avaliar a aplicabilidade do PTBC para a população brasileira, além de obter dados acerca da consistência interna desse checklist na população estudada.

\section{MÉTODO}

\section{Adaptação semântica para o português do PTBC}

Inicialmente, foi pedida a autorização ao autor do instrumento para a realização da tradução para o português e adaptação do instrumento para sua posterior utilização. Após concedida a autorização, dois profissionais de saúde bilíngues, um psiquiatra e outro psicólogo, fizeram a tradução do instrumento para o português do Brasil. As traduções foram feitas por cada um dos profissionais separadamente. Posteriormente, eles se reuniram e chegaram a uma versão em consenso.

Em seguida, foi realizada a retrotradução do instrumento, do português para o inglês. A retrotradução foi feita independentemente por dois professores de inglês bilíngues, um brasileiro e outro norte-americano, e nenhum dos dois conhecia o instrumento previamente. Posteriormente, os dois professores compararam as duas retrotraduções realizadas anteriormente e chegaram a uma versão retrotraduzida consensual.

No momento seguinte, a versão retrotraduzida consensual do PTBC foi avaliada pelo autor do instrumento, para que ele pudesse sugerir possíveis modificações, caso fossem necessárias. $\mathrm{O}$ autor avaliou a versão retrotraduzida consensual e não sugeriu nenhuma modificação, considerando que aquela versão poderia ser utilizada.

Sequencialmente, a versão avaliada pelo autor do instrumento foi preenchida por cinco mulheres, a fim de verificar se estas tinham uma boa compreensão de todos os itens. Como foi afirmado por todas elas que haviam compreendido bem todos os itens do instrumento, não se fez necessária modificação alguma e a versão utilizada tornou-se a versão final do instrumento.

\section{Aplicação do PTBC e avaliação da sua consistência interna}

Para a aplicação do PTBC, foi realizado um estudo de corte transversal, que ocorreu no período de janeiro a agosto de
2010, no qual se utilizou uma amostra de conveniência composta por 91 puérperas.

As mulheres foram abordadas aleatoriamente na recepção do ambulatório de puericultura do Hospital das Clínicas da Universidade Federal de Pernambuco (UFPE); as que preenchiam os critérios de inclusão foram convidadas a participar do estudo e conduzidas até uma das salas do ambulatório, onde eram entrevistadas por um dos pesquisadores responsáveis pelo estudo.

Os critérios de inclusão consistiam em: estar entre 2 e 26 semanas de pós-parto; aceitar participar voluntariamente do estudo e assinar o Termo de Consentimento Livre e Esclarecido; ser capaz de falar, ler e entender português; ter dado à luz um bebê tido por elas como saudável e, caso fossem menores de 18 anos, estar acompanhada de um responsável que lhe permitisse participar do estudo e assinasse o Termo de Consentimento Livre e Esclarecido.

Não puderam participar da pesquisa mulheres que estavam em surto psicótico, que não completaram a entrevista, que já tinham tido participação pregressa no estudo e que não preencheram os critérios de inclusão.

A pesquisa foi aprovada pelo Comitê de Ética em Pesquisa com Seres Humanos do Centro de Ciências da Saúde da UFPE, em 22 de maio 2009, sob protocolo n. ${ }^{\circ}$ 168/09.

As mulheres que participaram do estudo responderam à versão final do PTBC, uma entrevista semiestruturada que avalia a presença e o conteúdo de pensamentos intrusivos relacionados ao pós-parto, mais especificamente ao bebê, e as estratégias de neutralização utilizadas. Responderam também a um questionário biossociodemográfico elaborado pelos pesquisadores a fim de avaliar dados como: tempo de pós-parto, tipo de parto, possíveis complicações na gestação ou no parto, idade, estado civil, escolaridade e renda familiar da puérpera, assim como a presença de problemas de saúde e história pessoal e familiar de transtornos psiquiátricos. Ambos os instrumentos foram preenchidos pelos pesquisadores.

Para avaliar o grau de consistência interna do PTBC, foi obtido o alfa de Cronbach, que determina se a escala é realmente confiável, pois avalia como cada item reflete sua confiabilidade ${ }^{12}$.

\section{RESULTADOS}

\section{Tradução do PTBC}

A versão final da escala teve uma boa aceitação por parte das cinco primeiras puérperas entrevistadas. Essas não mencionaram dificuldades na compreensão ou interpretação e tampouco sugeriram modificações nos itens do checklist. Ao longo do estudo, foi observado que poucas vezes as puérperas solicitavam que algum dos itens fosse repetido e, quando isso ocorria, não era por dificuldade de compreensão do 
conteúdo, uma vez que, quando havia a repetição literal do que havia sido dito, elas respondiam. O tempo necessário para a aplicação do PTBC foi entre 10 e 15 minutos.

\section{Caracterização da amostra e avaliação da consistência interna do PTBC}

A amostra foi composta por 91 mulheres, a média de idade delas foi de aproximadamente 25 anos e o desvio-padrão correspondeu a 6 anos. Os dois graus de escolaridade mais frequentes corresponderam ao ensino médio $(35,2 \%)$ e ao ensino fundamental (27,5\%). No que se refere ao estado civil, os dois maiores percentuais foram: morava junto do companheiro $(40,7 \%)$ ou eram solteiras $(35,2 \%)$. Aproximadamente a metade $(49,5 \%)$ tinha renda até um salário-mínimo. Foi registrado que 17,6\% tinham problema de saúde, 12,1\% tinham histórico de transtorno psiquiátrico e 40,7\%, histórico familiar de transtorno psiquiátrico (Tabela 1).

Tabela 1. Caracterização da amostra, de janeiro a agosto de 2010, Recife (PE)

\begin{tabular}{|c|c|}
\hline Variável & Resultados \\
\hline Total & 91 \\
\hline Idade (anos): média \pm DP & $25,11 \pm 5,99$ \\
\hline Idade da criança (dias): média \pm DP & $84,51 \pm 50,77$ \\
\hline \multicolumn{2}{|l|}{ Escolaridade: $\mathrm{n}(\%)$} \\
\hline Ensino fundamental & $25(27,5)$ \\
\hline Ensino fundamental completo & $7(7,7)$ \\
\hline Ensino médio incompleto & $12(13,2)$ \\
\hline Ensino médio completo & $32(35,2)$ \\
\hline Ensino superior incompleto & $12(13,2)$ \\
\hline Ensino superior completo & $3(3,3)$ \\
\hline \multicolumn{2}{|l|}{ Estado conjugal: $n$ (\%) } \\
\hline Solteira & $32(35,2)$ \\
\hline Casada & $19(20,9)$ \\
\hline Mora junto do companheiro & $37(40,7)$ \\
\hline Separado & $3(3,3)$ \\
\hline \multicolumn{2}{|l|}{ Renda familiar: $\mathrm{n}(\%)$} \\
\hline Até 1 salário-mínimo & $45(49,5)$ \\
\hline Entre 2 e 5 salários & $43(47,3)$ \\
\hline Entre 6 e 10 salários & $2(2,2)$ \\
\hline Acima de 20 salários & $1(1,1)$ \\
\hline Pessoas dependentes da renda: Média \pm DP (mediana) & $4,20 \pm 1,43(4,00)$ \\
\hline \multicolumn{2}{|l|}{ Problemas de saúde: n (\%) } \\
\hline Sim & $16(17,6)$ \\
\hline Não & $75(82,4)$ \\
\hline \multicolumn{2}{|l|}{ Histórico de transtorno psiquiátrico: n (\%) } \\
\hline Sim & $11(12,1)$ \\
\hline Não & $80(87,9)$ \\
\hline \multicolumn{2}{|l|}{ Histórico familiar de transtorno psiquiátrico: $n$ (\%) } \\
\hline Sim & $37(40,7)$ \\
\hline Não & $54(59,3)$ \\
\hline
\end{tabular}

DP: desvio-padrão.
O coeficiente de consistência interna (alfa de Cronbach) do checklist, calculado após a aplicação do instrumento nas 91 puérperas, foi de 0,822.

\section{DISCUSSÃO}

Sabe-se que, para empregar instrumentos em uma cultura diferente da qual eles foram desenvolvidos, se faz necessária, além de sua tradução, uma adaptação que considere as circunstâncias sociais e culturais do local onde eles serão utilizados. A adaptação cultural de um instrumento psicométrico deve buscar o máximo de equivalência entre o instrumento original e sua versão traduzida, para, assim, evitar formas muitas vezes sutis de distorção, sendo assim um processo complexo. Um cuidado ainda maior deve existir quando o fenômeno a ser avaliado é subjetivo, como são os fenômenos psicopatológicos. Dessa maneira, o processo deve ser constituído por uma tradução conceitualmente equivalente ao original e culturalmente aceitável para o país onde será utilizado, sendo indispensável à busca por equivalência técnica e semântica entre as versões ${ }^{11}$.

No que se refere ao processo de tradução e adaptação do utilizado no presente estudo, avalia-se que a metodologia utilizada foi adequada, uma vez que não houve solicitação por parte do autor do instrumento para que nenhum item do instrumento retrotraduzido fosse modificado e que a versão final do instrumento pudesse ser facilmente compreendida pelas mulheres participantes do estudo.

Ao longo do estudo, foi observado que nos itens relacionados a agressividade/prejuízo/violência, assim como nos referentes a obsessões sexuais, as mulheres demonstravam espanto ou não respondiam, todavia foi observado que tal reação estava relacionada ao conteúdo expresso nos itens e não a uma dificuldade no entendimento do que estava sendo questionado.

O estudo foi desenvolvido com mulheres usuárias de um serviço da rede pública de saúde, em sua maioria com escolaridade até o Ensino Médio, o que consistiu numa limitação do estudo, uma vez que, dessa maneira, não se pode afirmar que o entendimento das questões seria o mesmo caso fossem avaliadas mulheres com diferentes níveis socioeconômicos e escolaridades. Todavia, ao longo do processo de tradução, houve uma cautela por parte dos profissionais para fazer uso de um vocabulário com ênfase no sentido literal das palavras; foram evitadas palavras rebuscadas ou expressões que caracterizassem uma parcela específica da população.

O preenchimento da escala foi feito pelo pesquisador; outra possibilidade de utilização seria o autopreenchimento. Todavia, devido ao baixo nível de escolaridade de algumas das mulheres entrevistadas, acredita-se que, caso a segunda opção fosse utilizada, haveria dificuldade por parte de algumas delas no preenchimento do instrumento. 
O coeficiente de consistência interna de um instrumento é medido pelo alfa de Cronbach. O objetivo da consistência interna é verificar se os itens possuem uma alta correlação, o que deveria ocorrer uma vez que estão medindo o mesmo constructo; dessa maneira, quanto mais homogêneo for o conteúdo expresso pelos itens, maior vai ser a consciência interna do instrumento ${ }^{12}$. O alfa de Cronbach é considerado alto quando está acima de 0,7. O PTBC apresentou um alfa total de 0,822 , o que pode ser considerado uma consistência interna muito boa.

Durante a aplicação da escala, foi percebido que um grande número das mulheres avaliadas apresentou pensamentos obsessivos em relação aos seus bebês e que tais pensamentos, por vezes, traziam sofrimento para elas. Percebeu-se ainda que, algumas vezes, estratégias comportamentais eram utilizadas pelas mulheres para neutralizar os pensamentos. Esses dados se mostram condizentes com os apresentados em estudos que utilizaram o PTBC, assim como com os que avaliaram pensamentos obsessivos no pós-parto ${ }^{3,6-8}$. A frequência e o conteúdo dos pensamentos apresentados pelas puérperas, assim como as estratégias de neutralização utilizadas por elas, serão tema de um artigo futuro.

Vale ressaltar que, como o PTBC permite ao profissional de saúde mental que acompanha a puérpera saber não apenas sobre seus pensamentos obsessivos em relação ao seu bebê, mas também quais são as estratégias que ela utiliza para neutralizar tais pensamentos e se isso acarreta dificuldade nos cuidados para com o bebê. Dessa maneira, a esse profissional, torna-se possível ter uma visão mais global de sua paciente, o que contribuirá para a elaboração de uma estratégia de tratamento mais eficaz.

É importante que pesquisas futuras possam trabalhar no desenvolvimento de instrumentos que avaliem não apenas a presença ou ausência de um pensamento ou comportamento, como ocorre em um checklist, mas de instrumentos que possam mensurar a intensidade e a frequência de tais pensamentos e comportamentos e relacioná-los ao grau de sofrimento apresentado pela puérpera.

\section{CONCLUSÕES}

Foi obtida a versão traduzida e adaptada para o português do Postpartum Thougths and Behavior Checklist, a qual teve a consistência interna avaliada, demonstrando-se ser muito boa. Dessa maneira, a versão obtida do PTBC pode ser considerada adequada para avaliar pensamentos obsessivos em relação ao bebê durante o puerpério na amostra em que o instrumento foi utilizado. Mais estudos são necessários para avaliar a efetividade da versão para o português do instrumento na população do Brasil.

\section{REFERÊNCIAS}

1. Maldonado MT. Psicologia da gravidez, parto e puerpério. 16. ed. São Paulo: Saraiva, 2002.

2. Camacho RS, Cantinelli FS, Ribeiro CS, Cantilino A, Gonsales BK, Braguittoni E, et al. Transtornos psiquiátricos na gestação e no puerpério: classificação, diagnóstico e tratamento. Rev Psiq Clín. 2006;33(2):92-102.

3. Abramowitz IS, Schwartz SA, Moore KM. Obsessional thoughts in postpartum females and their partners: content, severity, and relationship with depression. J Clin Psycholo Med. 2003;10(3):157-64

4. Abramowitz JS, Khandkes M, Nelson CA, Deacon BJ, Rygwall R. The role of cognitive factors in the pathogenesis of obsessive compulsive symptoms: A prospective study. Behav Res The. 2006;44(9):1361-74

5. Abramowitiz JS, Nelson CA, Rygwall R, Khandker M. The cognitive mediation of obsessive-compulsive symptoms: A longitudinal study. J Anxiety Disord. 2007;21(1):91-104.

6. Wisner KL, Peindl KS, Gigliotti T, Hanusa BH. Obsessions and compulsions in women with postpartum depression. J Clin Psychiatry. 1999;60(3):176-80.

7. Zambaldi CF. Sintomas obsessivo-compulsivos na depressão pós-parto [dissertação]. Recife (PE): Universidade Federal de Pernambuco, 2008.

8. Hall PL, Wittkowski A. An exploration of negative thougths as a normal phenomenon after childbirth. J Midwifery Womens Health. 2006;51(5):321-30.

9. Brockington IF, Macdonald E, Wainscott $G$. Anxiety, obsessions and morbid preoccupations in pregnancy and the puerperium. Arch. Womens Ment Health. 2006;9(5):253-63.

10. Goodman WK, Price LH, Rasmussen SA, Mazure C, Fleischman RL, Hill CL, et al. The Yale-Brown obsessive-compulsive scale: I. development use and reliability. Arch Gen Psychiatry. 1989;46(11):1006-11.

11. Streiner DL, Norman GR, editors. Health measurements scales: a pratical guide to their development and use. New York: Oxford University Press; 1995.

12. Maroco J, Garcia-Marques T. Qual a fiabilidade do Cronbach? Questões antigas e soluções modernas? Laboratório de Psicologia. 2006;4(1):65-90. 